\section{Gastrointestinal strongyloidiasis}
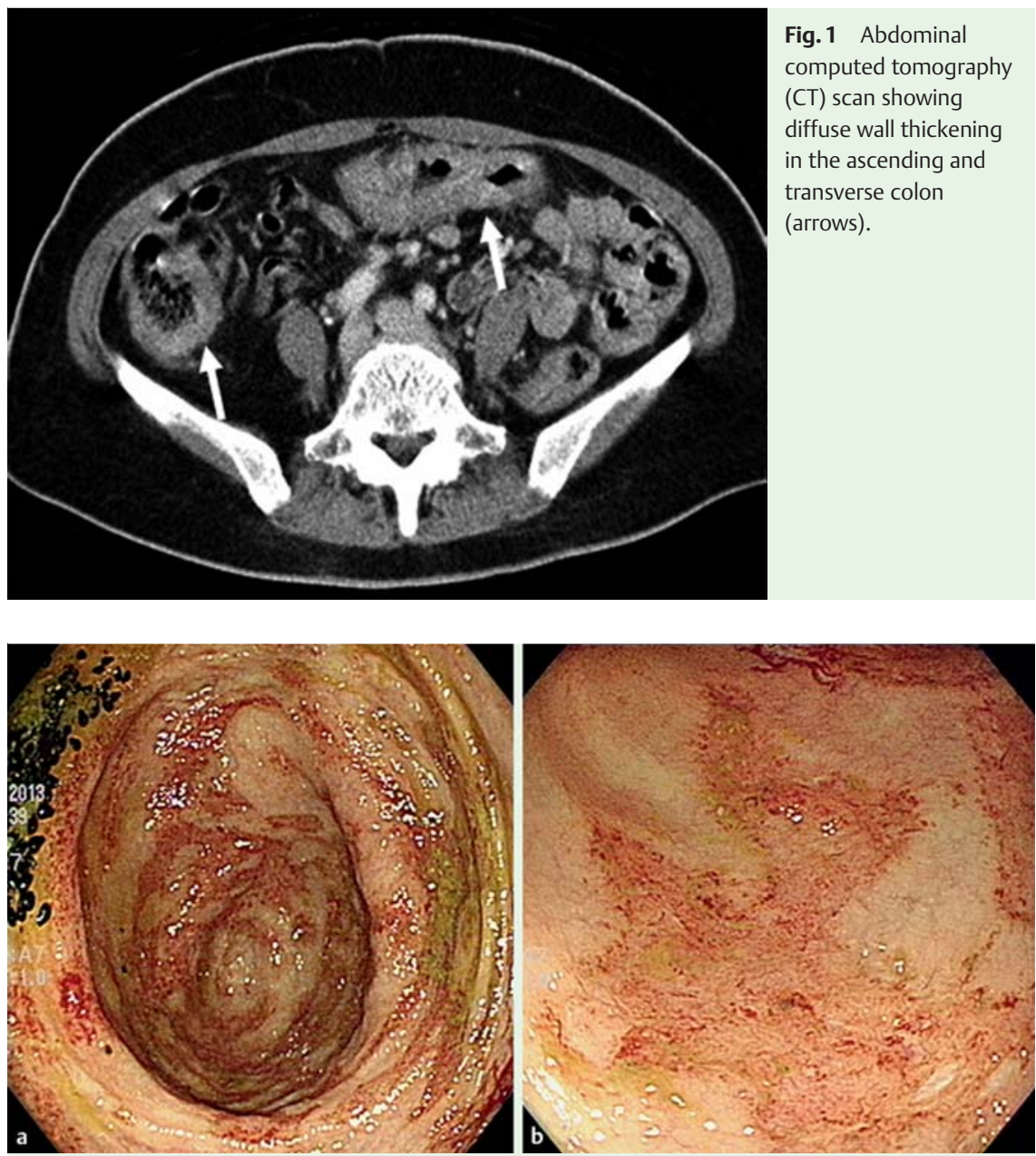

Fig. 2 Colonoscopy revealed diffuse hyperemic patches extending from the cecum to the transverse colon.

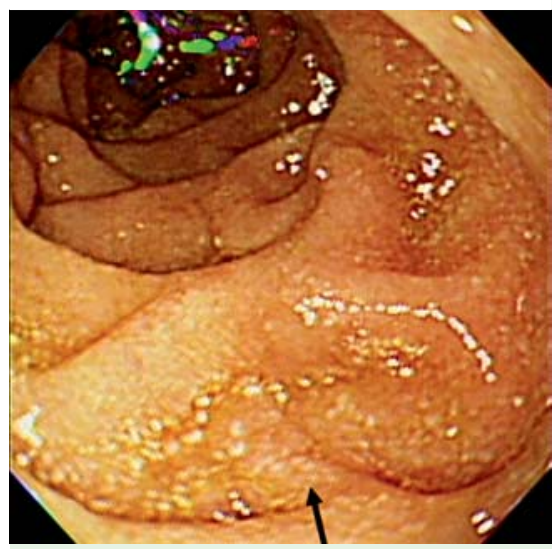

Fig. 3 Esophagogastroduodenoscopy revealed multiple tiny whitish nodules in the second portion of the duodenum (arrow).
A previously well, 62-year-old woman was referred to our emergency department with a 1-week history of fever, abdominal pain, and passing loose stool. On arrival, she was found on physical examination to have tenderness in the upper abdomen. The results of her laboratory tests showed a white blood cell count of $11.3 \times 10^{9} / \mathrm{L}$ (normal $3.5-11.0 \times 10^{9} / \mathrm{L}$ ) with eosinophils of $5.6 \%$ (normal $1 \%-5 \%$ ). Abdominal ultrasound and computed tomography (CT) scans revealed diffuse wall thickening of the ascending and transverse colon ( $\bullet$ Fig. 1). Colonoscopy showed diffuse hyperemic patches extending from the cecum to the transverse colon ( $\mathbf{F i g . 2}$ ). Esophagogastroduodenoscopy revealed multiple tiny whitish nodules in the second portion of the duodenum ( $\bullet$ Fig. 3 ).
Histopathological examination of biopsy specimens taken from the abnormal areas of the colon showed intact mucosa with filariform larvae of Strongyloides stercoralis in the lamina propria ( $\bullet$ Fig.4a). Biopsy specimens from the duodenum revealed adult female worms ( Fig. $\mathbf{4 b}, \mathbf{c}$ ) and rhabditiform larvae ( Fig. $\mathbf{4 b}$, d) of S. stercoralis in the crypts of the duodenal mucosa. Larvae of S. stercoralis were also found on examination of the stool.

The patient was therefore diagnosed as having gastrointestinal strongyloidiasis and she was treated with a single dose of ivermectin $(200 \mu \mathrm{g} / \mathrm{kg})$. Follow-up endoscopy 1 month later showed normal-looking mucosa in both the duodenum and colon, and histopathological examination of repeat biopsy specimens taken from duodenum and colon revealed no evidence of parasites.

The most common gastrointestinal symptoms of strongyloidiasis include nausea, vomiting, abdominal pain, and diarrhea $[1,2]$. The right-sided colon is involved in most patients. Endoscopic findings in strongyloidiasis are numerous and include loss of vascular pattern, erythema, erosions, ulcerations, and the presence of yellowish-white nodules [1,3].

In the normal life cycle, free-living filariform larvae enter the skin and the parasitic life cycle begins. The larvae enter the venous circulation, are transported to the lungs, penetrate the alveolar spaces, and are expectorated and swallowed. They arrive at the duodenum, where they mature into adult females and become embedded in the small-bowel mucosa. Here they lay embryonated eggs, from which rhabditiform larvae are released. The rhabditiform larvae migrate into the bowel lumen and travel down the intestines, passing out in the feces [4].

However, one important feature of some nematodes, including Strongyloides spp., is their ability to cause autoinfection. This means that the parasite never reaches the soil. Instead, the rhabditiform larvae develop into "infectious" filariform larvae in the bowel and directly infect the mucosa of the bowel, specifically the colon (endoautoinfection) and perianal skin area (exoautoinfection) [4].

Depending on its life cycle, different stages of S.stercoralis can be found in different parts of gastrointestinal tract [5]. As shown in this case, adult worms and rhabditiform larvae can be found in biopsy specimens of the duodenum and filariform 

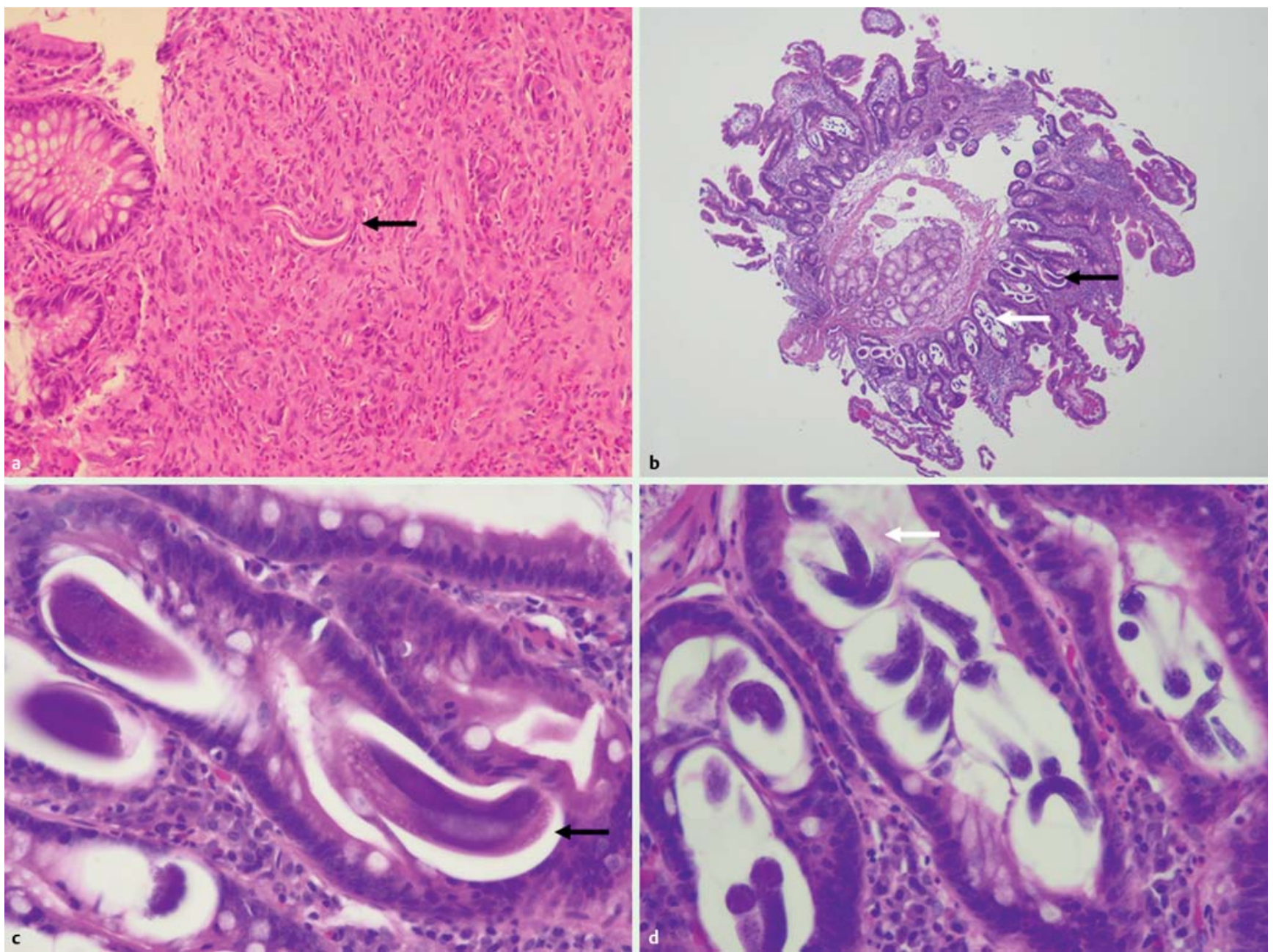

Fig. 4 Histopathological images of the hematoxylin and eosin (H\&E)-stained biopsy specimens taken from: a the involved colon showing filariform larvae of Strongyloides stercoralis (arrow) in the lamina propria (original magnification $\times 400$ ); $\mathbf{b}-\mathbf{d}$ the duodenum showing adult female worms (black arrow) and rhabditiform larvae (white arrow) of S. stercoralis in the crypts of the duodenal mucosa (original magnification: $\mathbf{b} \times 40$; $\mathbf{c}, \mathbf{d} \times 400$ ).

larvae can be found in biopsy specimens of the colon. Here, we have clearly demonstrated both the different endoscopic features and the different stages of the life cycle of $S$. stercoralis in a single case.

\section{Endoscopy_UCTN_Code_CCL_1AC_2AG}

\section{Competing interests: None}

\section{Chi-Jen Chen ${ }^{1}$, Jen-Wei Tsai ${ }^{2}$, Chi-Ming Tai ${ }^{1}$}

${ }^{1}$ Department of Internal Medicine, E-Da Hospital, I-Shou University, Kaohsiung, Taiwan

2 Department of Pathology, E-Da Hospital, I-Shou University, Kaohsiung, Taiwan

\section{References}

1 Thompson BF, Fry LC, Wells $C D$ et al. The spectrum of GI strongyloidiasis: an endoscopic-pathologic study. Gastrointest Endosc 2004; 59: 906-910

2 Wang C, Xu J, Zhou X et al. Strongyloidiasis: an emerging infectious disease in China. Am J Trop Med Hyg 2013; 88: 420 - 425

3 Minematsu H, Hokama A, Makishi T et al. Colonoscopic findings and pathologic characteristics of Strongyloides colitis: a case series. Digestion 2011; 83: 210-214

4 Kassalik M, Mönkemüller K. Strongyloides stercoralis hyperinfection syndrome and disseminated disease. Gastroenterol Hepatol (N Y) 2011; 7: 766 - 768

5 Schär F, Trostdorf U, Giardina F et al. Strongyloides stercoralis: global distribution and risk factors. PLoS Negl Trop Dis 2013; 7: e2288

\section{Bibliography}

DOI http://dx.doi.org/ 10.1055/s-0034-1365386

Endoscopy 2015; 47: E187-E188

(c) Georg Thieme Verlag KG

Stuttgart · New York

ISSN 0013-726X

\section{Corresponding author}

\section{Chi-Ming Tai, MD}

Department of Internal Medicine

E-Da Hospital

1, E-Da Rd

Jiau-Shu Village

Yan-Chau District

Kaohsiung 824

Taiwan (ROC)

Fax: +886-7-6155352

chimingtai@gmail.com 